\title{
Genetic Variability and Geographical Distribution of Mycotoxigenic Fusarium verticillioides Strains Isolated from Maize Fields in Texas
}

\author{
Carlos S. Ortiz ${ }^{1}$, Casey Richards ${ }^{2}$, Ashlee Terry ${ }^{2}$, Joselyn Parra ${ }^{2}$ and Won-Bo Shim ${ }^{1,2 *}$ \\ ${ }^{I}$ Department of Plant Pathology and Microbiology, Texas A\&M University, College Station, TX 77843-2132, USA \\ ${ }^{2}$ Bioenvironmental Sciences Program, Texas A\&M University, College Station, TX 77843-2132, USA
}

(Received on February 12, 2015; Revised on April 22, 2015; Accepted on April 29, 2015)

Maize is the dominant cereal crop produced in the US. One of the main fungal pathogens of maize is Fusarium verticillioides, the causative agent of ear and stalk rots. Significantly, the fungus produces a group of mycotoxins - fumonisins - on infested kernels, which have been linked to various illnesses in humans and animals. Nonetheless, durable resistance against $F$. verticillioides in maize is not currently available. In Texas, over 2.1 million acres of maize are vulnerable to fumonisin contamination, but understanding of the distribution of toxigenic $F$. verticillioides in maize-producing areas is currently lacking. Our goal was to investigate the genetic variability of $F$. verticillioides in Texas with an emphasis on fumonisin trait and geographical distribution. A total of $164 F$. verticillioides cultures were isolated from 65 maize-producing counties. DNA from each isolate was extracted and analyzed by PCR for the presence of FUM1- a key fumonisin biosynthesis gene - and mating type genes. Results showed that all isolates are in fact $\boldsymbol{F}$. verticillioides capable of producing fumonisins with a 1:1 mating-type gene ratio in the population. To further study the genetic diversity of the population, isolates were analyzed using RAPD fingerprinting. Polymorphic markers were identified and the analysis showed no clear correlation between the RAPD profile of the isolates and their corresponding geographical origin. Our data suggest the toxigenic $F$. verticillioides population in Texas is widely distributed wherever maize is grown. We also hypothesize that the population is fluid, with active movement and genetic recombination occurring in the field.

Keywords : Fusarium verticillioides, population, fumonisin, RAPD, Texas

*Corresponding author.

Phone) 1-979-458-2190, FAX) 1-979-845-6983

E-mail)wbshim@tamu.edu
Maize, one of the leading cereal crops in the world, yields a versatile commodity not only used for food and feed, but also in a wide range of industrial products. According to the US Department of Agriculture National Agricultural Statistics Service, maize is the main agricultural crop in the US with more than 90.6 million acres harvested in 2014 and more than 14.2 billion bushels produced. While maize is not considered the main crop produced in Texas, growers planted over 2.1 million acres of land yielding more than 294 million bushels of grain in 2014. According to the Food and Agriculture Organization (FAO) of the United Nations (http://www.fao.org), 25\% of the world's food crops are affected by mycotoxins representing global losses of food products in the range of 1 billion tons per year.

Fusarium verticillioides (teleomorph Gibberella moniliformis) is one of the most devastating pathogens of maize (Bacon and Nelson, 1994; Munkvold and Desjardins, 1997). The fungus is capable of causing ear rot and stalk rot diseases, frequently without causing early visible external symptoms (Munkvold and Desjardins, 1997; Smith and White, 1988). The fungus is a heterothallic ascomycete and can rapidly reproduce and disperse via asexual spores, e.g., macroconidia and microconidia, on infested seeds and plant debris (Desjardins, 2003; Leslie and Summerell, 2006). Recently, attention to crop diseases caused by $F$. verticillioides has drastically increased due to a group of mycotoxins, fumonisins, produced by the fungus on infested grains (Gelderblom et al., 1988; Marasas et al., 2001). Fumonisin B1 (FB1), the predominant fumonisin found in nature, is highly toxic to human and animals when ingested and has been linked to esophageal cancer and neural tube defect in humans as well as numerous illnesses in animals (Gelderblom et al., 1988; Marasas et al., 2001; Nelson et al., 1993). Environmental factors such as temperature, drought stress and humidity levels are known to be associated with FB1 production while dry and cool storage conditions are known to deter further production of the toxin (Bacon and Nelson, 1994; Shelby et al., 1994). 
When severe kernel damage is found, strategies to manage the infection are similar to those of other ear rot diseases and usually comprise the readjustment of the combine and subsequent cleaning, drying and proper storage of the grain (Munkvold and Desjardins, 1997). Industry guidance levels for fumonisin were released in 2001 by the United States Food and Drug Administration (2001) to establish the maximum fumonisin levels permitted in human foods and animal feeds (http://www.fda.gov). The levels are set between 2-4 ppm in different maize products directed to human consumption while higher levels, ranging from 5 to $100 \mathrm{ppm}$, are set for animal feeds depending on the animal class.

Direct genetic resistance to $F$. verticillioides is currently not available to growers. However, the use of transgenic maize hybrids, such as $B t$ maize, has provided moderate relief (Munkvold et al., 1999). Maize infection by $F$. verticillioides often occurs by the landing of airborne or rainsplashed inoculum into wounded plant tissue, and insects are known to play an important role in creating wounds. These insects include the European corn borer (Ostrinia nubilalis), corn earworm, corn rootworm and Western flower thrips (Munkvold and Desjardins, 1997). Bt maize harbors cry genes from the soil bacterium Bacillus thuringiensis that produce insecticidal crystalline proteins in plant tissue (Hofte and Whiteley, 1989). These toxins ultimately lead to decreased insect feeding and thus results in less F. verticillioides colonization of maize kernels and stalks (Munkvold et al., 1999).

With the lack of durable maize resistance to $F$. verticillioides and FB1 contamination, understanding the genetic diversity and distribution of $F$. verticillioides in the field could provide useful information for developing better region-specific disease management practices. Unfortunately, such data for maize producing counties in Texas is currently lacking. Molecular markers based on the polymerase chain reaction (PCR) can be used to quickly study the genetic diversity of organisms. One of the techniques often used is random amplified polymorphic DNA (RAPD) where a single primer of arbitrary nucleotide sequence is used to detect genetic variations in a given population by amplifying DNA regions (polymorphism) of individual members (Williams et al., 1990). Such polymorphisms are heritable changes in a genome that arise by mutations (Clark, 1976) and thus provide a DNA fingerprint of an organism. These fingerprints, or genetic markers, can then be used in to analyze the genetic fluidity and diversity within a species. Published reports show successful application of RAPD techniques in $F$. verticillioides, with primers readily available (da Silva et al., 2006; Desjardins and Plattner,
2000; Moller et al., 1999).

The goal of our study was to investigate the genetic variability of $F$. verticillioides in Texas with an emphasis on fumonisin B1 trait and geographical distribution. Here, we isolated $F$. verticillioides field samples from sixty-five maize-producing counties in Texas and subsequently performed RAPD analyses. Additionally, we tested the sexual mating alleles and the genetic potential of the strains to produce FB1 toxin.

\section{Materials and Methods}

Maize sample collection. Texas maize samples from the 2011 harvest were obtained by the Office of the Texas State Chemist, following the One Sample Strategy (Office of the Texas State Chemist, 2012). Briefly, $2.2 \mathrm{~kg}$ maize samples per truck were collected at approved grain elevators, probing maize loads 7-to-9 times with a $72 \mathrm{~cm}$ spiral hand probe using representative sampling patterns described previously (Federal Crop Insurance Corporation, 2010). Once collected, the entire $2.2 \mathrm{~kg}$ sample was ground in a Romer mill (Romer Labs) until at least 70\% of the particles pass through a No. 20 wire woven mesh sieve. Using a spatula, the ground sample was then stirred for 30 $\mathrm{s}$ to ensure a homogenous blend and $50 \mathrm{~g}$ subsamples were weighed, placed and marked in plastic bags. Bags were stored at $-20^{\circ} \mathrm{C}$ until further studies were conducted.

Fungal cultures isolation. A minimum of three bags of pulverized maize per county were randomly chosen when sufficient samples were available. Pulverized maize (100 $\mathrm{mg}$ ) was transferred to a tube containing sterile water (1 $\mathrm{ml}$ ) and vortexed for $10 \mathrm{~s}$. We further diluted the sample by transferring $10 \mu \mathrm{l}$ of the solution into a new tube containing $1 \mathrm{ml}$ of sterile water. The diluted sample $(50 \mu \mathrm{l})$ was then spread onto petri dishes containing $0.2 \times$ potato dextrose agar (PDA) medium amended with ampicillin and streptomycin $(50 \mathrm{mg} / \mathrm{ml})$ and incubated for four days at room temperature. This process was repeated with each pulverized maize bag. Once fungal growth was observed, individual colonies were isolated, subcultured as a single microconidium, transferred to individual petri dishes and incubated at room temperature. Isolates exhibiting the standard F. verticillioides phenotype (Leslie and Summerell, 2006) were further analyzed. At least three independent isolates per county, when possible, were used in this study. $F$. verticillioides wild-type strains 7600 (MAT1-1) and 7598 (MAT1-2) (Fungal Genetics Stock Center) as well as our isolated $F$. verticillioides strains were stored in $30 \%$ glycerol (v/v) at $-80^{\circ} \mathrm{C}$ in this study. 
DNA extraction from fungal cultures. Yeast extract peptone dextrose (YEPD) broth $(5 \mathrm{ml})$ was poured into a petri dish $(60 \times 15 \mathrm{~mm})$ and inoculated with an agar plug $(0.5 \mathrm{~cm}$ in diameter) containing fungal mycelium. Plates were incubated at room temperature without agitation for 5 days. Once time lapsed, the broth was vacuumed out and the fungal tissue was flash frozen in liquid nitrogen. DNA was extracted using the OmniPrep kit (G-Biosciences) following the manufacturer's recommended protocol. DNA concentrations were determined using a NanoDrop 2000 system (Thermo Fisher Scientific) and DNA quality was visually inspected by gel electrophoresis. DNA working solutions $(150 \mathrm{ng} / \mu \mathrm{l})$ were used for all PCR in this study unless otherwise stated.

Polymerase chain reaction. All PCR analyses were performed using a $25 \mu \mathrm{l}$ final aliquot volume of the amfiEco Taq DNA Polymerase kit (Gendepot) following the manufacturer's recommendation in either a GeneAmp PCR system 9700 (Applied Biosystems). Amplification of mating type genes (MAT1-1 and MAT1-2) as well as the FUM1 gene was carried out using primer pairs listed in Table 1. All three sets of PCR were carried out at $52^{\circ} \mathrm{C}$ annealing temperature and $1 \mathrm{~min}$ extension for 35 cycles. RAPD markers were produced with a single primer (Table 1) with an annealing temperature of $35^{\circ} \mathrm{C}$ and $2 \mathrm{~min}$ extension. The final concentration of all primers was 15 pmol. Electrophoresis of standard PCR amplicons and RAPD products was carried out in standard $1 \%$ and $1.5 \%$ agarose gels in $0.5 \times$ TBE buffer, respectively, with subsequent ethidium bromide staining/destaining and photography under UV light. DNA samples from wild-type strains, $F$. verticillioides 7600 and 7598, were used as controls.

Cluster analyses of genetic markers. The RAPD profile of each isolate was obtained by comparing their individual marker pattern to the patterns of all the samples across the population produced with the same primer. Variations in band intensity of such markers was disregarded and a binomial system, where 1 is positive and 0 is negative, was used to score for the presence or absence of markers generated with a given primer. Once the OPA-01, OPB01 and UBC-18-generated profiles of all the samples were scored, the 3 profiles per isolate were consolidated into one in an OPA-OPB-UBC fashion. Using the DendromUPGMA utility (http://genomes.urv.cat/UPGMA/) described by Garcia-vallvé et al. (1999), a similarity matrix of the RAPD profiles was computed using Dice similarity coefficient, 100 bootstrap replicates and cluster analysis was performed using an unweighted pair-group method using an arithmetic averaging (UPGMA) algorithm. A dendrogram was then drawn using Phylogeny (http://www.phylogeny. fr) (Dereeper et al., 2008). Geographical maps of the State of Texas were generated using the diymaps utility (http:// diymaps.net/tx.htm).

FB1 assay. One g of cracked maize was placed in glass vials, rehydrated in water overnight and then autoclaved. Each vial was then independently inoculated with $1 \times 10^{6}$ spores of $F$. verticillioides wild-type strain 7600 and strains Bailey \#140B, Floyd \#61B, Bailey \#141B, Castro \#72, Parmer \#22B, Lamb \#135B, Dallam \#70, Milam \#217, Moore \#237A or Hale \#29A isolated from Texas panhandle area counties. Vials were then placed in an incubator for 10 days at $24^{\circ} \mathrm{C}$ with a $14 \mathrm{~h}$ light $/ 10 \mathrm{~h}$ dark cycle. Subsequently, extraction of FB1 and ergosterol was accomplished by adding $5 \mathrm{ml}$ of acetonitrile:water $(1: 1, \mathrm{v} / \mathrm{v})$ and chloroform:methanol $(2: 1, \mathrm{v} / \mathrm{v})$, respectively, to the vials and incubated at room temperature overnight without agitation. Purification of extracts was performed following protocols described by Shim and Woloshuk (1999) where FB1 crude extracts (acetonitrile:water vials) are passed through equilibrated HyperSep C18 SPE columns (Thermo Fisher Scientific) and subsequently washed with water and 15\%

Table 1. Primers used in this study

\begin{tabular}{lll}
\hline Name & Sequence $\left(5^{\prime}-3-\right)$ & Reference \\
\hline Gfmat1a & GTTCATCAAAGGGCAAGCG & Steenkamp et al., 2000 \\
Gfmat1b & TAAGCGCCCTCTTAACGCCTTC & Steenkamp et al., 2000 \\
Gfmat2c & AGCGTCATTATTCGATCAAG & Steenkamp et al., 2000 \\
Gfmat2d & CTACGTTGAGAGTGTACAG & Steenkamp et al., 2000 \\
FvFum-F & TCACGAACCCTTGAAAGCAG & Shim and Woloshuk, 2001 \\
FvFum-R & TACAAATTGTCCACGAGGCG & Shim and Woloshuk, 2001 \\
UBC-18 & GGGCCGTTTA & Möller et al., 1999 \\
OPA-01 & CAGGCCCTTC & Desjardins and Plattner, 2000 \\
OPB-01 & GTTTCGCTC & Desjardins and Plattner, 2000 \\
\hline
\end{tabular}


acetonitrile and then eluted in $70 \%$ acetonitrile. On the other hand, ergosterol crude extracts (chloroform:methanol vials) were passed over Acrodisc $13 \mathrm{~mm}$ nylon $0.45 \mathrm{~mm}$ filters (Pall Life Sciences). HPLC analyses of FB1 and ergosterol were performed as described elsewhere (Kim et al., 2011; Shim and Woloshuk, 1999). FB1 and ergosterol concentrations were quantified by comparing the peak area to that of FB1 and ergosterol standards (25 ppm), respectively. FB1 levels were then normalized to fungal growth using Kim et al. (2011) equation where FB1 biosynthesis $=[\mathrm{Fb} 1$ production $(\mu \mathrm{g} / \mathrm{g}) /$ ergosterol contents $(\mu \mathrm{g} / \mathrm{g})] \times 100$. Three biological replicates were performed per sample. Standard error was calculated based on the average values of wild type replicates and the average values of the 10 field isolates.

\section{Results}

$F$. verticillioides is ubiquitous in Texas maize production fields. Maize samples from 65 counties, representing $24.8 \%$ of the total counties in the state (254 counties), were analyzed for the presence of $F$. verticillioides. We isolated $164 F$. verticillioides strains using standard identification techniques described previously (Leslie and Summerell, 2006). Cultures exhibited white aerial mycelia growing in uniform radial form and developed pigments ranging from light pink to violet gray with age. We isolated at least 3 samples from $50.7 \%$ of the counties surveyed while we were able to isolate one or two samples in $49.2 \%$ of the counties. Samples from some counties $(13.8 \%$ of the total) yielded 4 to $7 F$. verticillioides isolates.

Both $F$. verticillioides mating types are present and randomly dispersed throughout Texas. Out of the 164 fungal isolates tested, 82 contained the MAT1-1 mating type locus while the remaining 82 tested positive for the MAT12 locus, therefore presenting an equal mating-type distribution in all isolates tested. All isolates from Dallam, Gray, Hartley, Hidalgo, Hill, Hutchinson, Swisher and Victoria counties corresponded to the MAT1-1 mating type while all those from Hale, Live Oak, Medina, Wharton and Wilson counties were only positive for the MAT1-2 mating type. The remaining 51 counties showed mixed mating-type distribution (Fig. 2b). However, it is important to note that this outcome does not indicate that one mating-type dominates certain counties since we tested a small population sample of $F$. verticillioides isolates from each county.

All $F$. verticillioides samples isolated in this study were positive for the FUM1 gene, which encodes a polyketide synthase critical for fumonisin biosynthesis. The FUM1
A

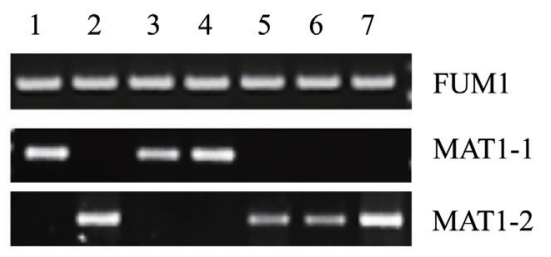

B

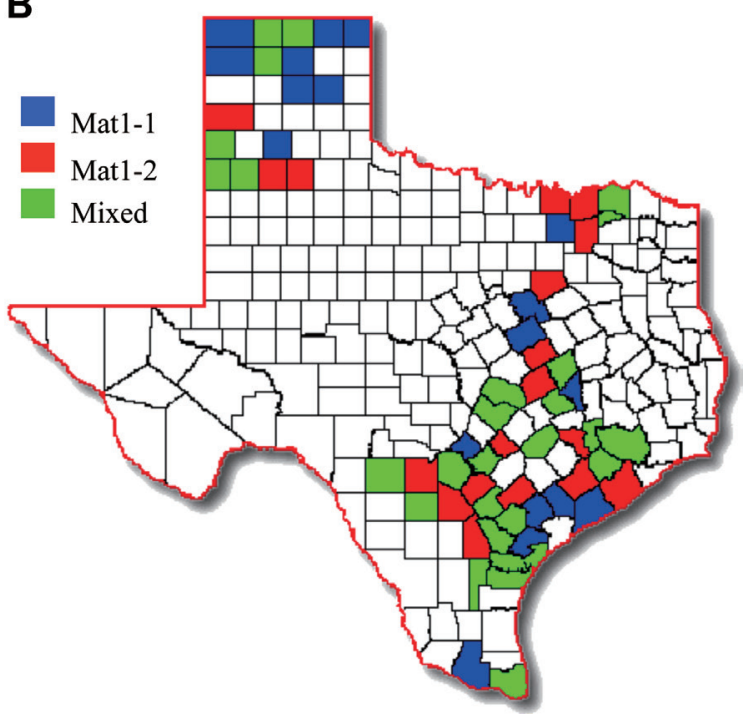

Fig. 1. Mating type and fumonisin-producing capability in F. verticilioides strains of Texas. (A) PCR of the mating-type loci genes and FUM1 gene in $164 F$. verticillioides field isolates and reference strains (7600 and 7598). Results were analyzed by electrophoresis on a 1.0\% agarose gel stained with ethidium bromide. Lane 1, Wild-type strain 7600 (MAT1-1); Lane 2, Wild-type strain 7598 (MAT1-2); Lane 3-7, isolates Victoria \#1, Waller \#2, Falls \#38, Medina \#240A and Robertson \#242A, respectively. (B) Isolate mating type was color-coded and graphed onto a Texas map based on the county of origin for all isolates. Counties colored blue and red represent counties where all samples isolated were MAT1-1 and MAT1-2, respectively. Green counties are those where mixed mating-type populations were observed. 
A

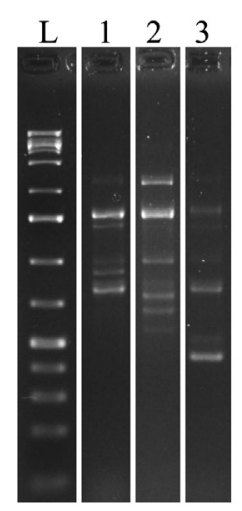

OPA-01
B

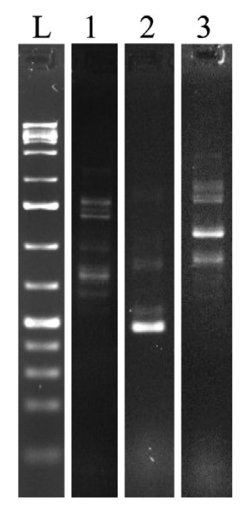

OPB-01
C

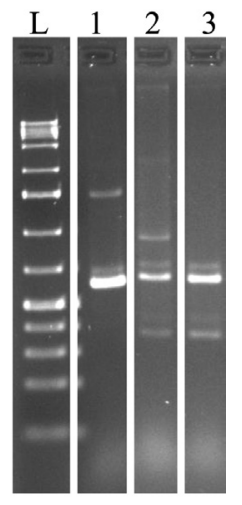

UBC-18

Fig. 2. Random amplified polymorphic DNA (RAPD) of field isolates. Representative samples of markers generated via PCR with OPA-01, OPB-01 and UBC-18 primers. Samples were subjected to electrophoresis in a $1.5 \%$ agarose gel and stained with ethidium bromide. Lane $\mathrm{L}$ is showing DNA ladder. Wild type strains 7600, 7598 and isolate Dallam \#70 in lane 1, 2 and 3, respectively. (A) Ten markers were generated with the OPA-01 primer. (B) Eleven distinctive markers were produced with the OPB-01 primer. (C) Six different markers resulted from the PCR reaction with the UBC-18 primer.

gene is the central component of the FUM gene cluster and is often used as a diagnostic marker for fumonisinproducing Fusarium species (Shim and Woloshuk, 2001; Shim et al., 2003). This result suggests that all $F$. verticillioides isolates found in the Texas maize production fields are capable of producing fumonisins under environmental conditions that favor mycotoxin biosynthesis.

Polymorphic markers reveal fluid $F$. verticillioides diversity within Texas maize fields. Using RAPD techniques, while disregarding variations in band intensity, we identified 27 polymorphic markers using 3 different primers previously utilized for $F$. verticillioides diagnostics (Desjardins and Plattner, 2000; Moller et al., 1999). Ten markers were generated when using the OPA-01 primer, eleven markers with the OPB-01 primer and six markers with the UBC-18 primer (Fig. 3). Polymorphisms were scored by the presence or absence of a given marker resulting in a binomial DNA fingerprint. We used the unweighted pair group method with arithmetic mean (UPGMA) with the Dice coefficient (Garcia-Vallvé et al., 1999) to analyze the RAPD fingerprints and cluster the samples based on similarity. We then generated a dendrogram which was essential for determining the clustering patterns.

In order to observe the relationship between the RAPD

profile data and the geographic point of origin of each sample, we color-coded the clusters of Texas counties into six regions (Fig. 4a), which corresponds to the map of the Texas Health Service regions. Strains in the dendrogram were then color-coded to reflect their region of origin (Fig. $4 b$ ). The three samples originating from Burleson County clustered together. This trend was also observed with all samples originating from Dallam, Goliad and Fort Bend Counties (Fig. 4a-2), but neither of the counties grouped together. Clusters of two samples per county were also observed in McLennan, Lamb, Frio, Lamar, Brazoria, Moore, Uvalde, Comal, Bell and Medina counties. Finally, mating type data was added onto the dendrogram (data not shown) and, similar to earlier results, no clear correlation between RAPD profile and geographic location was observed.

F. verticillioides strains isolated from the Texas panhandle region are capable of producing fumonisin $\mathrm{B} 1$. Ten $F$. verticillioides cultures isolated from maize samples from Texas Panhandle counties were randomly selected for in vitro FB1 production assays. Notably, in the 2011 annual report published by the Office of the Texas State Chemist, maize samples collected from this region did not show detectable levels of fumonisins (http://mycotoxinbmps. tamu.edu/mapsupdate.aspx). Thus, we tested whether $F$. verticillioides strains we isolated from the region's maize samples were non-toxigenic or they simply failed to produce fumonisins due to unfavorable environmental conditions. Our results showed that FB1 was produced by all samples tested with detection values ranging from 62.313 (Bailey \#140B) to 183.312 ppm (Dallam \#70) while $F$. verticillioides 7600 produced $50.603 \mathrm{ppm}$. FB1 values were normalized to endogenous ergosterol levels, compounded and graphed (Fig. 4).

\section{Discussion}

More than 2 million acres of maize are planted and harvested every year in the State of Texas with most of this production concentrated in 65 counties (Fig. 1). F. verticillioides is one of the most common pathogens of maize worldwide and is known to produce mycotoxins, such as fumonisins, which has been linked to human and animal illnesses. In the late 1980's and early 1990's increased incidences of leukoencephalomalacia in horses and neural tube defects in human newborns were detected in South Texas (Hendricks, 1999; Marasas et al., 2003). This lead to a casefinding study where the association of neural tube defect and consumption of fumonisin-tainted corn tortillas in Cameron county and other Texas-Mexico border coun- 

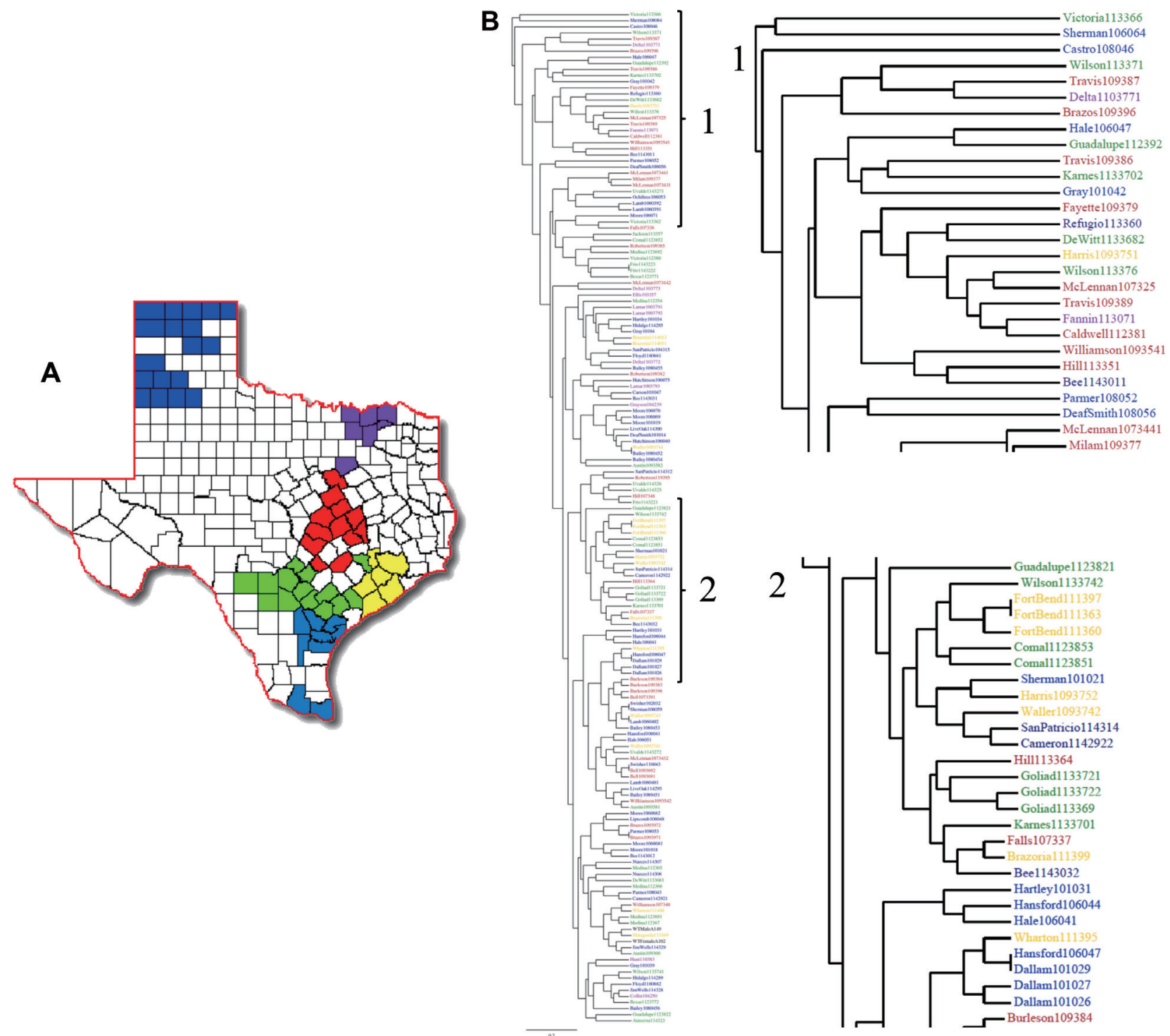

Fig. 3. Genetic variability of F. verticillioides strains in Texas. (A) Maize producing counties of Texas were color coded and grouped into 6 different geographical regions. These are the 65 counties where most of the 2.1 million acres of maize production is concentrated. (B) A dendrogram was produced with the RAPD profiles of the $F$. verticillioides field isolates. Each sample was then color-coded representing their county of origin.

ties, was hypothesized (Missmer et al., 2006).

Historically, the presence of $F$. verticillioides was assumed to be less of a concern in the southern United States since weather conditions were not considered favorable for infection and fumonisin production when compared to conditions found in the Midwest. However, due to devastating health-related predicaments and subsequent diligent monitoring by the US FDA, maize growers and producers have become increasingly aware of the issues associated with fumonisin contamination in food and feeds. Notably, recent surveys performed by the OTSC show increased and persistent levels of fumonisins in Texas maize (Li, 2012).
As mentioned earlier, commercial hybrids with effective resistance to $F$. verticillioides are not currently available to growers, and the management of Fusarium ear rot relies on the use of hybrids with physical traits, e.g., Bt transgene and tightly enclosed husks protecting the silk channels, that minimizes pathogen transmission. Other management practices, e.g. early harvesting, improved postharvest storage, and rotation with non-host crops, are also recommended. However, in order to implement these management practices, growers must be aware of pathogenic and epidemiological potential of $F$. verticillioides isolates in the maize production regions. 


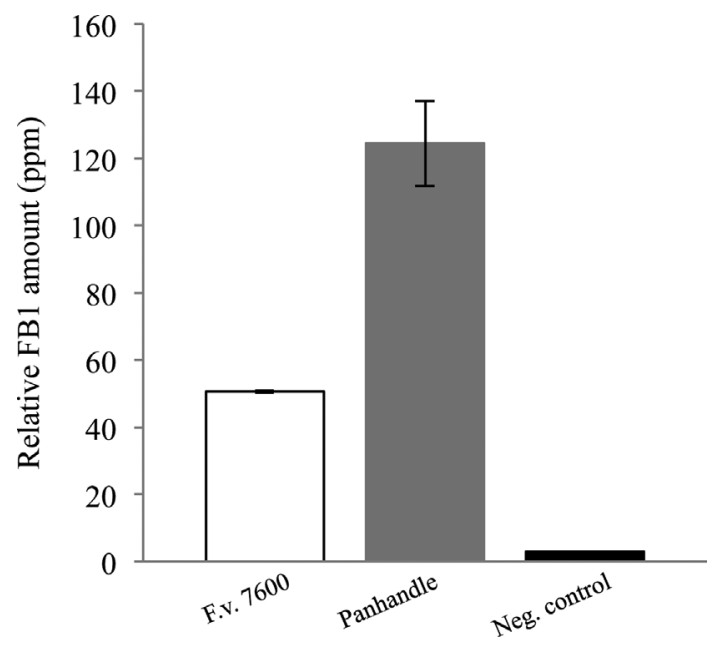

Fig. 4. Fumonisin B1 production by $F$. verticillioides strains isolated from Texas Panhandle counties. In vitro FB1 production was normalized to endogenous ergosterol levels. Standard error bars were calculated by the average FB1 production levels of 10 panhandle isolates or FB1 levels of replicates of the wild-type strain 7600. Negative control is FB1 extracted from autoclaved maize kernels inoculated with sterile water.

In this study we analyzed $164 F$. verticillioides strains isolated from the 65 maize-producing counties in Texas. Fumonisins, and especially FB1, are known to be produced mainly by two Fusarium species, $F$. verticillioides and $F$. proliferatum. Sequence-specific primers for FUM1, the gene encoding the structural backbone and start of the biosynthetic pathway of fumonisins, have been designed and are routinely used to confirm the genetic capability to produce fumonisins (Shim et al., 2003; Shim and Woloshuk, 2001). All of our isolates tested positive when screened for the presence of the FUM1 gene. According to the Office of the Texas State Chemist data, no FB1 was detected in 2011 maize samples originated from Texas Panhandle counties. To test the FB1 biosynthesis potential of $F$. verticillioides strains isolated from such area, we randomly selected 10 strains and inoculated maize kernels in vitro. Our data showed that all strains tested are capable to synthesize FB1 at levels similar (50 ppm) or higher (up to 4 times more) than that of $F$. verticillioides wild-type strain 7600 . We presented the data as a compounded graph of all isolates compared to the wild-type strain (Fig. 4) in order to highlight a regional trend. In our water control, we were able to detect trace amounts of FB1, and this could be attributed to innate FB1 contamination in the commercial cracked maize blending we used. No mycelial growth was observed in these samples. FB1 levels in water control are presented for disclosure. Based on our FUM1 screening assay and in vitro $\mathrm{FB} 1$ production data, it is reasonable to conclude that
F. verticillioides isolates in Texas are genetically capable of producing FB1 under conducive field conditions.

Sexual reproduction increases the ability of an organism to genetically recombine and increase the genetic diversity in offspring. In fungi, sexual compatibility between haploid individuals is determined by a small chromosomal region known as the mating-type locus (MAT) (Fraser and Heitman, 2004; Fraser and Heitman, 2005). F. verticillioides is a heterothallic organism with two recognized mating-type idiomorphic alleles, MAT1-1 and MAT1-2, in the MAT1 locus. Our results showed a 1:1 mating type ratio with no clear geographical clustering of mating types observed in areas tested. $F$. verticillioides is recognized as a seed and soilborne pathogen, and its spores can be randomly dispersed by wind or splashing of water (Munkvold and Desjardins, 1997). Such mobility of inoculum could explain the lack of clear mating-type clustering in a restricted region. In addition, this random distribution of the two mating types suggests that active sexual recombination is occurring in the maize production fields across the State. Further experiments are warranted to test this hypothesis.

In order to investigate the diversity of $F$. verticillioides in Texas maize fields, we conducted genetic analyses of the 164 strains using RAPD techniques. Through the employment of three primers, we retrieved and scored twenty seven polymorphic bands in total. Genetic similarity analyses (Garcia-Vallvé et al., 1999) were performed using Dice coefficient and UPGMA algorithms. In order to corroborate the analysis results, a second analysis was also performed using Jaccard coefficient instead of the Dice coefficient without resulting in dendrogram topology changes other than branch length differences. Both Jaccard and Dice coefficients are considered to be adequate for the study of genetic divergence employing RAPD techniques (Duarte et al., 1999). The resulting dendrograms showed little, if any, clustering of samples based on region of origin or mating type suggesting a fluid population of $F$. verticillioides in Texas. Interestingly, fourteen counties showed two or more samples to segregate together. This could be due to the possibility of clonal isolates, a small pool of polymorphic markers or markers not specific enough to distinguish between highly similar samples. We recognize the limitations of RAPD, particularly in reproducibility, and that techniques such as RFLP, AFLP and next-generation sequencing are available for more accurate and precise population genetic studies. However, in this study we were able to clearly determine the genetic variability in our isolates with RAPD and arrive at our conclusion that there is no recognizable pattern in $F$. verticillioides population in Texas.

Extreme climate, e.g. the drought Texas experienced in 
2012, and increased levels of fumonisins in Texas maize pose new challenges to the safekeeping of foods and feeds. A better understanding of the distribution and population of $F$. verticillioides in maize fields may provide an advantage when designing and pursuing new and improved disease management practices. To our knowledge, this is the first report of a compilation of $F$. verticillioides strains isolated from Texas maize fields and the study of their genetic variability.

\section{Acknowledgments}

This work was supported by funding from the Alfred P. Sloan Foundation Minority Ph.D. Program, Department of Plant Pathology and Microbiology-Texas A\&M University, and The Hispanic Serving Institutions Education Grants Program (\#TEXE-2006-03485) to C. Ortiz and from the Louis Stokes Alliance for Minority Participation program at Texas A\&M University to C. Richards. The authors would like to acknowledge Dr. Tim Herrman, Director of the Office of the Texas State Chemist, for providing the maize samples, and Drs. Clint Magill and Heather Wilkinson for their critical insight on the $F$. verticillioides population analysis.

\section{References}

Bacon, C. W. and Nelson, P. E. 1994. Fumonisin production in corn by toxigenic strains of Fusarium moniliforme and $\mathrm{Fu}$ sarium proliferatum. J. Food Protect. 57:514-521.

Clark, W. C. 1976. The environment and the genotype in polymorphism. Zool. J. Linn. Soc.-Lond. 58:255-262.

da Silva, V. N., Fernandes, F. M. C., Cortez, A., Ribeiro, D. H. B., de Almeida, A. P., Hassegawa, R. H. and Corrêa, B. 2006. Characterization and genetic variability of Fusarium verticillioides strains isolated from corn and sorghum in Brazil based on fumonisins production, microsatellites, mating type locus, and mating crosses. Can. J. Microbiol. 52:798-804.

Dereeper, A., Guignon, V., Blanc, G., Audic, S., Buffet, S., Chevent, F., Dufayard, J. F., Guindon, S., Lescot, M., Claverie, J. M. and Gascuel, O. 2008. Phylogeny.fr: robust phylogenetic analysis for the non-specialist. Nucleic Acids Res. 36:W465-W469.

Desjardins, A. E. and Plattner, R. D. 2000. Fumonisin B1-nonproducing strains of Fusarium verticillioides cause maize (Zea mays) ear infection and ear rot. J. Agr. Food Chem. 48:57735780.

Desjardins, A. E. 2003. Gibberella from $A$ (venaceae) to $Z$ (eae). Annu. Rev. Phytopathol. 41:177-198.

Duarte, J. M., Santos, J. B. D. and Melo, L. C. 1999. Comparison of similarity coefficients based on RAPD markers in the common bean. Genet. Mol. Biol. 22:427-432.
Federal Crop Insurance Corporation. 2010. Loss Adjustment Manual (LAM) Standards Handbook: 2010 and succeeding crop years Washington, DC. United States Departmet of Agriculture.

Food and Agriculture Organization of the United Nations. Mycotoxins. In: FAO.org. [http://www.fao.org/food/food-safetyquality/a-z-index/mycotoxins/en/]. Accessed 29 March 2013.

Food and Drug Administration. 2001.Guidance for industry: Fumonisin levels in human foods and animal feeds; Final guidance. Rockville, MD. United States Department of Health and Human Services.

Fraser, J. A. and Heitman, J. 2004. Evolution of fungal sex chromosomes. Mol. Microbiol. 51:299-306.

Fraser, J. A. and Heitman, J. 2005. Chromosomal sex-determining regions in animals, plants and fungi. Curr. Opin. Genet. Dev. 15:645-651.

Garcia-Vallvé, S., Palau, J. and Romeu, A. 1999. Horizontal gene transfer in glycosyl hydrolases inferred from codon usage in Escherichia coli and Bacillus subtilis. Mol. Biol. Evol. 16:1125-1134.

Gelderblom, W. C. A., Jaskiewicz, K., Marasas, W. F. O., Thiel, P. G., Horak, R. M., Vleggaar, R. and Kriek, N. P. J. 1988. Fumonisins - novel mycotoxins with cancer-promoting activity produced by Fusarium moniliforme. Appl. Environ. Microbiol. 54:1806-1811.

Hendricks, K. 1999. Fumonisins and neural tube defects in South Texas. Epidemiology 10:198-200.

Hofte, H. and Whiteley, H. R. 1989. Insecticidal crystal proteins of Bacillus thuringiensis. Microbiol. Rev. 53:242-255.

Kim, H., Smith, J. E., Ridenour, J. B., Woloshuk, C. P. and Bluhm, B. H. 2011. HXK1 regulates carbon catabolism, sporulation, fumonisin B1 production and pathogenesis in Fusarium verticillioides. Microbiol-SGM 157:2658-2669.

Leslie, J. F. and Summerell, B. A. 2006. The Fusarium laboratory manual. Oxford: Blackwell Publishing.

Li, W., Dai, S. Y. and Herrman, T. 2012. Mycotoxin analysis in animal feed by LC/MS/MS. Office of the Texas State Chemist, College Station.

Marasas, W. F. O., Miller, J. D., Riley, R. T. and Visconti, A. 2001. Fumonisins-occurrence, toxicology, metabolism and risk assessment. Fusarium: Paul E Nelson Memorial Symposium, 332-359.

Missmer, S. A., Suarez, L., Felkner, M., Wang, E., Merrill, A. H., Rothman, K. J. and Hendricks, K. A. 2006. Exposure to fumonisins and the occurrence of neural tube defects along the Texas-Mexico border. Environ. Health Persp. 114: 237-241.

Möller, E. M., Chelkowski, J. and Geiger, H. H. 1999. Speciesspecific PCR assays for the fungal pathogens Fusarium moniliforme and Fusarium subglutinans and their application to diagnose maize ear rot disease. J. Phytopathol. 147:497-508.

Munkvold, G. P. and Desjardins, A. E. 1997. Fumonisins in maize: Can we reduce their occurrence? Plant Dis. 81:556565.

Munkvold, G. P., Hellmich, R. L. and Rice, L. G. 1999. Compari- 
son of fumonisin concentrations in kernels of transgenic Bt maize hybrids and nontransgenic hybrids. Plant Dis. 83:130138.

National Agricultural Statistics Service. 2012. 2011 Crop production. Washington, DC. Agricultural Statistics Board of the United States Department of Agriculture.

Nelson, P. E., Desjardins, A. E. and Plattner, R. D. 1993. Fumonisins, mycotoxins produced by Fusarium species: biology, chemistry, and significance. Ann. Rev. Phytopathol. 31:233252.

Office of the Texas State Chemist. 2012. One sample strategy for aflatoxin risk management in Texas. College Station, TX. Texas A\&M University System.

Shelby, R. A., White, D. G. and Bauske, E. M. 1994. Differential fumonisin production in maize hybrids. Plant Dis. 78:582584.

Shim, W. B. and Woloshuk, C. P. 1999. Nitrogen repression of fumonisin B1 biosynthesis in Gibberella fujikuroi. FEMS Microbiol. Lett. 177:109-116.

Shim, W. B. and Woloshuk, C. P. 2001. Regulation of fumonisin
B1 biosynthesis and conidiation in Fusarium verticillioides by a cyclin-like (C-type) gene, FCC1. Appl. Environ. Microbiol. 67:1607-1612.

Shim, W. B., Flaherty, J. E. and Woloshuk, C. P. 2003. Comparison of fumonisin $\mathrm{B} 1$ biosynthesis in maize germ and degermed kernels by Fusarium verticillioides. J. Food Protect. 66:2116-2122.

Smith, D. R. and White, D. G. 1988. Diseases of corn. In: Sprague, G.F. and Dudley, J.W. eds. Corn and Corn Improvement. Madison, WI: American Society of Agronomy, pp. 687766.

Steenkamp, E. T., Wingfield, B. D., Coutinho, T. A., Zeller, K. A., Wingfield, K. J., Marasas W. F. O. and Leslie J. F. 2000. PCR-based identification of MAT-1 and MAT-2 in the Gibberella fujikuroi species complex. Appl. Environ. Microbiol. 66:4378-4382.

Williams, J. G. K., Kubelik, A. R., Livak, K. J., Rafalski, J. A. and Tingey, S. V. 1990. DNA polymorphisms amplified by arbitrary primers are useful as genetic-markers. Nucleic Acids Res. 18:6531-6535. 